\title{
Fatores de risco para histerectomia em mulheres brasileiras
}

\author{
Risk factors for hysterectomy \\ among Brazilian women
}

Thália V. Barreto de Araújo 1

Estela M. L. Aquino 2

\footnotetext{
1 Departamento de Medicina Social, Universidade Federal de Pernambuco. Av. Professor Morais Rego s/n, Hospital das Clínicas, 4o andar, Bloco E, Cidade Universitária, Recife, $P E$ 50670-420, Brasil. thalia@br.inter.net 2 MUSA - Programa de Estudos em Gênero e Saúde, Instituto de Saúde Coletiva, Universidade Federal da Bahia. Rua Padre Feijó 29, 4o andar, Salvador, BA 40110-170, Brasil. musa@ufba.br
}

\begin{abstract}
A case-control study was conducted to investigate risk factors for hysterectomy among women using the public health system in Northeast Brazil. The cases were 373 women aged 30 54 years that had undergone elective hysterectomy for benign pelvic conditions. Controls were 742 women with preserved uterus selected from public health clinics. Data were collected through a review of medical records and a personal interview using a structured, pre-tested questionnaire. Unconditional multiple logistic regression was applied in the analysis. Women at greater risk for hysterectomy were those with a higher per capita family income, zero to three children, a history of medical consultation for menstrual problems, hospitalization for gynecological problems, or tubal ligation before age 30 years. Menopause and a history of stillbirth appeared as protective factors in the statistical analysis.
\end{abstract}

Key words Hysterectomy; Women's Health; Reproductive Health; Case-control Studies

Resumo Realizou-se um estudo de caso-controle exploratório para investigar fatores de risco para histerectomia entre usuárias do setor público de saúde em Recife, Pernambuco, Brasil. Os 373 casos são mulheres com idade entre 30 e 54 anos, submetidas à histerectomia eletiva por condição pélvica benigna. Os controles, 742 mulheres com útero preservado, foram selecionados em centros de saúde. Os dados foram coletados em entrevistas, com um questionário estruturado e pré-testado, ou transcritos dos prontuários médicos. Com base em regressão logística múltipla não-condicional, foram identificados como fatores de risco para histerectomia: a renda familiar per capita relativamente maior, a nuliparidade e a paridade de até três filhos, a demanda por cuidados médicos por problemas menstruais, o antecedente de distúrbio menstrual ou de morbidade do colo uterino, a hospitalização por causa ginecológica e a presença de laqueadura tubária antes dos trinta anos. A história prévia de filho nascido morto e a menopausa se constituíram em fatores de proteção.

Palavras-chave Histerectomia; Saúde da Mulher; Saúde Reprodutiva; Estudos de Casos e Controle 


\section{Introdução}

A histerectomia consiste na remoção cirúrgica do útero. As informações disponíveis não permitem estimar a prevalência deste procedimento entre mulheres brasileiras. Todavia, no Sistema Único de Saúde (SUS), a histerectomia é a segunda cirurgia mais freqüente entre mulheres em idade reprodutiva, sendo precedida apenas pelo parto cirúrgico. Em 1999, foram realizadas 93.597 histerectomias em unidades hospitalares do SUS (DATASUS, 1999). À semelhança do que ocorre em outros países (Treloar et al., 1999; Wilcox et al., 1994), a grande maioria é indicada por enfermidades benignas (DATASUS, 1999). No ano citado, as neoplasias malignas representaram $5,1 \%$ das histerectomias realizadas no país e $16,7 \%$ daquelas realizadas em Recife (DATASUS, 1999).

As comparações internacionais mostram grandes discrepâncias nos níveis, tendência e padrões de indicação de histerectomia entre os países (Settnes \& Jorgensen, 1996; Wilcox et al., 1994), e entre áreas de um mesmo país (Roos, 1984). As pesquisas que enfocaram os fatores que predispõem as mulheres à realização da histerectomia mostram que as taxas de ocorrência dessa cirurgia podem elevar-se em função de características sociodemográficas, do padrão reprodutivo (em especial, com o aumento da idade e da paridade) e de certas práticas contraceptivas - uso do dispositivo intra-uterino ou de contraceptivos orais por longo prazo, e após laqueadura tubária (Brett et al., 1997; Goldhaber, 1993; Treloar et al., 1999).

Alguns estudos evidenciam que o contexto sociocultural no qual se realizam as práticas de saúde voltadas às mulheres pode influenciar a freqüência de realização da cirurgia. Assim, a possibilidade de acesso aos serviços de saúde (Roos, 1984), a filiação a plano privado de assistência (Haas et al., 1993), as características do provedor das ações de saúde (Luoto et al., 1997) e dos médicos consultados, as atitudes e crenças destes médicos com respeito ao procedimento, como também, o desejo da mulher em evitar a cirurgia (Bickell et al., 1994; Domenighetti et al., 1988), são fatores que podem explicar parte da variação observada no risco de histerectomia entre mulheres de uma mesma população.

Este estudo teve por objetivo investigar fatores de risco associados à histerectomia entre usuárias do SUS em Recife, capital do Estado de Pernambuco, buscando-se conhecer elementos que participam na configuração dessa prática no âmbito do setor público de saúde.

\section{Metodologia}

Realizou-se um estudo de caso-controle exploratório, sediado em cinco grandes hospitais do SUS. Dos hospitais selecionados, dois são gerenciados pelo Estado, dois são hospitais de ensino universitário e o outro é privado, conveniado ao SUS.

\section{Definição e seleção dos casos} e dos controles

Os casos representam todas as mulheres, residentes em Recife, com idades entre 30 e 54 anos, submetidas à histerectomia eletiva nos hospitais selecionados, entre 4 de abril de 1999 e 3 de abril de 2000, identificados por meio dos registros das cirurgias e das admissões ao internamento. Foram inelegíveis aquelas em que a cirurgia ocorreu associada à gravidez ou resultou de neoplasia maligna, sendo os prontuários médicos e laudos histopatológicos revistos para confirmação. Os controles são mulheres, também residentes em Recife, que referiram ter o útero preservado, selecionadas de forma aleatória nos ambulatórios de clínica médica dos centros de saúde nos quais os casos foram diagnosticados e as cirurgias indicadas. Foram inelegíveis as gestantes, puérperas e aquelas que informaram o diagnóstico prévio de câncer ginecológico. Os casos foram pareados aos controles por freqüência pela idade, em faixas de cinco anos, na razão de 2:1.

\section{Produção dos dados}

\section{e categorização das variáveis}

Os dados provêem de entrevistas face a face para a aplicação de questionário estruturado e pré-codificado. Estas foram realizadas nas unidades de saúde, sendo os casos entrevistados após a cirurgia ainda no período de hospitalização. Foram coletadas informações sobre potenciais fatores de risco para histerectomia, incluindo-se características sócio-demográficas; história menstrual, reprodutiva e de morbidade ginecológica; uso de contracepção e de serviços de saúde.

Para a categorização do padrão menstrual prevalente ao início da vida adulta (em torno dos vinte anos) e daquele predominante nos cinco anos que antecederam a cirurgia, em ciclos sem ou com transtorno menstrual, foram utilizados os parâmetros: dor menstrual, número de dias de sangramento, regularidade e duração do ciclo menstrual, como descritos pelas entrevistadas. Maiores detalhes encontramse em outro local (Barreto-de-Araújo, 2002). 


\section{Análise dos dados}

A seleção das variáveis para inclusão na análise multivariada foi baseada na revisão da literatura específica e nos dados empíricos, sendo incluídas na modelagem aquelas variáveis cuja associação com o efeito estudado, na análise bivariada, atingiu nível de significância estatística igual ou inferior a 0,20 (valor de p). Na análise de regressão logística múltipla não condicional utilizou-se o procedimento backward, sendo as variáveis selecionadas para exclusão do modelo com base no nível de significância para a razão de máxima verossimilhança (RMV). Para a avaliação de significância estatística das associações encontradas, foram obtidos o intervalo de confiança a $95 \%$ e o valor p. Nesta etapa, as variáveis categóricas (que apresentaram anteriormente ponto de corte definido), foram transformadas em dicotômicas. A renda foi dicotomizada utilizando-se o valor mediano, que correspondeu à metade do salário mínimo em vigência na época da pesquisa $(\mathrm{R} \$ 136,00)$.

Inicialmente, as variáveis foram divididas em dois grupos, tendo por referência a literatura sobre o tema: um composto por variáveis que expressavam processos ou eventos biológicos modelo biológico: nunca ter engravidado, ter tido filho natimorto, ter tido hospitalização por causa ginecológica, ter realizado cirurgia ginecológica prévia, referir distúrbio menstrual aos vinte anos, relatar antecedente de morbidade do colo do útero, ter história de demanda por cuidados médicos por sangramento uterino, e relatar o uso da pílula por cinco anos ou mais; e o outro, por variáveis que representavam características das mulheres com maior potencial para influir na decisão médica quanto à indicação da histerectomia - modelo motivacional: nunca ter vivido em união, participação na força de trabalho, vida sexual ativa, paridade, menopausa, laqueadura tubária, idade na última gestação e renda per capita familiar. No processo de modelagem, a idade das mulheres foi introduzida como variável contínua. O processamento para a análise estatística foi efetuado utilizando-se o programa SPSS-PC versão 8.00.

\section{Resultados}

Foram entrevistadas 1.115 mulheres, sendo 373 casos e 742 controles. Dentre os casos elegíveis houve dois óbitos $(0,5 \%)$, oito perdas $(2,1 \%) \mathrm{e}$ quatro recusas à participação (1,0\%). Entre os controle elegíveis houve quatro recusas $(0,5 \%)$.

Em todas as cirurgias, o procedimento adotado foi a histerectomia total, que em $24,1 \%$ dos casos esteve associada à salpingo-ooforectomia bilateral. Nos casos em que os dois ovários foram removidos, 94,4\% não apresentaram anormalidades ao exame histopatológico. Quanto ao padrão de indicação, o diagnóstico de leiomioma uterino - tumor benigno das fibras musculares do útero - foi responsável por 91,9\% das cirurgias.

Dado ao pareamento de freqüência aplicado à idade, a distribuição por grupo etário foi idêntica entre os dois grupos. A tabela 1 sumariza as características sócio-demográficas para os casos e os controles. O fato das mulheres terem ou não companheiro ou vida sexual ativa à época da pesquisa não influenciou o risco de histerectomia (Tabela 1).

Os principais resultados sobre a história reprodutiva e contraceptiva são apresentados na Tabela 2. Dentre as mulheres que realizaram histerectomia há uma proporção maior que nunca teve filhos ou engravidou. Contudo, o risco de histerectomia não aumentou de forma linear em função do número de partos (teste para tendência linear $\mathrm{p}=0,240$, excluída a paridade zero). As características das entrevistadas diferem dentro de cada um dos níveis de paridade (Tabela 3).

Quanto às perdas fetais, a ocorrência de natimorto foi relatada com mais freqüência pelos controles do que pelos casos; porém, não foram observadas diferenças quanto à história de abortamentos (Tabela 2). Para os abortos espontâneos, provocados ou o total destes, o ajuste pelo efeito da paridade aproximou ainda mais os resultados da hipótese nula (valores de $\mathrm{p}>0,20$ ).

O padrão de uso de métodos contraceptivos reafirmou a predominância da pílula e da laqueadura tubária sobre as demais formas de regulação da fecundidade no Brasil. Na etapa inicial da análise, não ficou evidenciada uma associação entre laqueadura tubária e histerectomia (Tabela 2), mas, ao ajuste pelo efeito da paridade, variável identificada como confundidora para a associação, o risco de histerectomia foi maior entre as mulheres com laqueadura tubária do que entre aquelas sem laqueadura. As mulheres que usaram pílula por cinco anos ou mais, tiveram um risco de histerectomia mais elevado (OR = 1,46; IC95\%: 1,02-2,09; $\mathrm{p}=$ 0,0299), em comparação àquelas que usaram a pílula por período mais curto.

A Tabela 4 apresenta as variáveis relativas à história menstrual e de morbidade ginecológica, e a freqüência de uso de serviços de ginecologia. A existência de alteração do padrão menstrual foi a razão pela qual iniciou-se a investigação clínica que determinou a indicação da histerectomia em $51,7 \%$ dos casos. 
Tabela 1

Características sócio-demográficas dos casos de histerectomia e dos controles.

\begin{tabular}{|c|c|c|c|c|c|c|c|}
\hline \multirow[t]{2}{*}{ Características } & \multicolumn{2}{|c|}{ Casos } & \multicolumn{2}{|c|}{ Controle } & \multicolumn{3}{|c|}{ OR não ajustada } \\
\hline & $n$ & $\%$ & $n$ & $\%$ & OR & IC $95 \%$ & valor-p \\
\hline \multicolumn{8}{|l|}{ Idade (anos completos) 1} \\
\hline $30-34$ & 34 & 9,1 & 68 & 9,2 & & 1 & \\
\hline $35-39$ & 74 & 19,8 & 148 & 19,9 & & & \\
\hline $40-44$ & 101 & 27,1 & 202 & 27,2 & & & \\
\hline $45-49$ & 111 & 29,8 & 219 & 29,5 & & & \\
\hline $50-54$ & 53 & 14,2 & 105 & 14,2 & & & \\
\hline \multicolumn{8}{|l|}{ Situação conjugal } \\
\hline Nunca esteve & 36 & 9,7 & 42 & 5,7 & 1,69 & $1,03-2,77$ & 0,0027 \\
\hline Já esteve & 73 & 19,7 & 175 & 23,8 & 0,82 & $0,60-1,14$ & 0,2196 \\
\hline Está em união & 262 & 70,7 & 517 & 70,4 & 1,00 & 2 & \\
\hline \multicolumn{8}{|l|}{ Presença de companheiro } \\
\hline Nunca teve & 21 & 5,6 & 27 & 3,6 & 1,53 & $0,82-2,87$ & 0,2015 \\
\hline $\mathrm{N}$ ão tem & 90 & 24,1 & 198 & 26,7 & 0,90 & $0,66-1,21$ & 0,4626 \\
\hline Tem & 262 & 70,2 & 517 & 69,7 & 1,00 & 2 & \\
\hline \multicolumn{8}{|l|}{$\begin{array}{l}\text { Inserção no mercado } \\
\text { de trabalho } 3\end{array}$} \\
\hline $\operatorname{Sim}$ & 225 & 60,3 & 375 & 39,7 & 1,49 & $1,15-1,93$ & 0,0020 \\
\hline Não & 148 & 50,5 & 367 & 49,5 & 1,00 & 2 & \\
\hline \multicolumn{8}{|l|}{$\begin{array}{l}\text { Renda familiar mensal } \\
\text { per capita (em Reais) }\end{array}$} \\
\hline 68,17 e + & 208 & 62,7 & 280 & 43,7 & 2,16 & $1,63-2,86$ & 0,0000 \\
\hline A té 68,17 reais & 124 & 37,3 & 361 & 56,3 & 1,00 & 2 & \\
\hline \multicolumn{8}{|l|}{ Escolaridade 4} \\
\hline $\mathrm{N}$ ão freqüentou escola & 37 & 10,0 & 86 & 11,8 & 1,00 & 2 & \\
\hline Ensino básico & 167 & 68,7 & 328 & 69,4 & 1,18 & $0,76-1,86$ & 0,5063 \\
\hline Ensino fundamental & 118 & 10,8 & 231 & 10,9 & 1,19 & $0,74-1,93$ & 0,5184 \\
\hline Ensino médio ou mais & 39 & 10,5 & 58 & 7,9 & 1,56 & $0,77-2,18$ & 0,1541 \\
\hline
\end{tabular}

1 Variável submetida ao pareamento de freqüência, por isso não inclui o cálculo de OR.

2 Categoria de referência.

3 Refere-se aos doze meses que antecederam a cirurgia.

4 Estão incluídas nas categorias as mulheres que cursaram uma ou mais séries correspondentes ao grau especificado.

$O R=0 d d s$ ratio.

\section{Análise multivariada}

No modelo biológico foi identificada uma associação positiva entre histerectomia e presença de distúrbios menstruais aos vinte anos, demanda por cuidados médicos por problemas menstruais, antecedente de morbidade (referida) do colo uterino, hospitalização por causa ginecológica e já ter engravidado. A história pregressa de gravidez que resultou em natimorto mostrou um efeito protetor (Tabela 5).

Permaneceram no modelo motivacional como fatores de risco para histerectomia, a nuliparidade e a paridade de até três filhos, a presença de laqueadura tubária e a renda familiar mensal per capita relativamente maior. Estar na menopausa persistiu como fator de proteção (Tabela 5).

Nesta etapa da análise, o risco de histerectomia não se mostrou associado ao uso prolongado (cinco anos ou mais) de contraceptivo oral ( $p=0,1223$ ), à ocorrência de cirurgia ginecológica prévia ( $\mathrm{p}=0,3951$ ), à ausência de vida sexual ativa ( $p=0,2186)$, ao exercício de atividade produtiva ( $\mathrm{p}=0,1018$ ), ao fato da mulher nunca ter vivido em união $(\mathrm{p}=0,1649)$ ou à idade das mulheres na última gestação ( $\mathrm{p}=0,3445$ ), tendo sido estas variáveis excluídas dos respectivos modelos. 
Características dos casos de histerectomia e controles segundo história sexual, reprodutiva e contraceptiva.

\begin{tabular}{|c|c|c|c|c|c|c|}
\hline \multirow[t]{2}{*}{ Características } & \multicolumn{2}{|c|}{ Casos $(n=373)$} & \multicolumn{2}{|c|}{ Controles $(n=742)$} & \multicolumn{2}{|c|}{ OR não-ajustada } \\
\hline & $\%$ & $\mathrm{n}$ & $\%$ & $\mathrm{n}$ & OR (IC95\%) & valor-p \\
\hline \multicolumn{7}{|l|}{ Vida sexual ativa } \\
\hline Nunca teve & 4,3 & 16 & 3,0 & 22 & $0,78(0,58-1,05)$ & 0,0860 \\
\hline $\mathrm{N}$ ão tem & 24,7 & 92 & 30,0 & 221 & $1,36(0,67-2,74)$ & 0,3596 \\
\hline Sim & 71,0 & 26 & 67,0 & 494 & 1 & \\
\hline \multicolumn{7}{|l|}{ Número de gestações } \\
\hline Nenhuma & 12,6 & 47 & 8,0 & 59 & $1,77(1,16-2,71)$ & 0,0083 \\
\hline 1 & 5,6 & 21 & 8,0 & 59 & $0,79(047-1,35)$ & 0,3884 \\
\hline 2 & 18,8 & 70 & 15,7 & 116 & $1,34(0,95-1,90)$ & 0,0983 \\
\hline 3 & 18,8 & 70 & 18,9 & 140 & $1,11(0,79-1,56)$ & 0,5410 \\
\hline 4 e mais & 44,2 & 165 & 49,5 & 367 & 1 & \\
\hline Média (desvio-padrão) & 3,69 & 2,89 & 4,18 & 3,21 & (Mann Whitney, & $=0,0180)$ \\
\hline Número de filhos tidos (paridade) & & & & & & 0,0036 \\
\hline Nenhum & 14,5 & 54 & 8,8 & 66 & $2,10(1,35-3,28)$ & 0,0004 \\
\hline 1 & 8,3 & 31 & 10,6 & 78 & $1,02(0,62-1,68)$ & 0,9263 \\
\hline 2 & 24,1 & 90 & 21,3 & 158 & $1,47(1,03-2,09)$ & 0,0273 \\
\hline 3 & 23,6 & 88 & 21,2 & 157 & $1,44(1,01-2,06)$ & 0,0354 \\
\hline 4 e mais & 29,5 & 110 & 38,1 & 283 & 1 & \\
\hline Média (desvio-padrão) & 3,01 & 2,36 & 3,47 & 2,66 & (Mann Whitney, & $=0,004)$ \\
\hline \multicolumn{7}{|l|}{ Número total de abortos } \\
\hline 1 e mais & 33,0 & 123 & 37,1 & 275 & $0,84(0,0,64-1,10)$ & 0,2014 \\
\hline Nenhum & 67,0 & 250 & 62,9 & 467 & 1 & \\
\hline \multicolumn{7}{|l|}{ Abortos espontâneos } \\
\hline 1 e mais & 23,9 & 89 & 28,8 & 214 & $0,77(0,57-1,04)$ & 0,0905 \\
\hline Nenhum & 76,1 & 284 & 71,2 & 528 & 1 & \\
\hline \multicolumn{7}{|l|}{ Abortos induzidos } \\
\hline 1 e mais & 13,7 & 51 & 11,5 & 85 & $1,22(0,83-1,80)$ & 0,3317 \\
\hline Nenhum & 86,3 & 322 & 88,5 & 657 & 1 & \\
\hline \multicolumn{7}{|l|}{ Filhos nascidos mortos } \\
\hline 1 e mais & 4,8 & 18 & 8,2 & 61 & $0,57(0,32-1,00)$ & 0,0372 \\
\hline Nenhum & 95,2 & 355 & 91,8 & 681 & 1 & \\
\hline \multicolumn{7}{|l|}{ Idade na primeira gravidez } \\
\hline$<15$ & 7,1 & 23 & 5,8 & 39 & $1,65(0,89-3,06)$ & 0,1114 \\
\hline $15-19$ & 43,0 & 139 & 42,8 & 289 & $1,35(0,91-2,00)$ & 0,1406 \\
\hline $20-24$ & 35,9 & 116 & 32,8 & 222 & $1,46(0,97-2,20)$ & 0,0674 \\
\hline 25 e mais & 13,9 & 45 & 18,6 & 126 & 1 & \\
\hline \multicolumn{7}{|l|}{ Idade na última gravidez } \\
\hline$<19$ & 4,3 & 14 & 3,3 & 22 & $2,04(0,86-4,82)$ & 0,1145 \\
\hline $20-24$ & 24,1 & 78 & 19,5 & 131 & $1,91(1,12-3,26)$ & 0,0154 \\
\hline $25-29$ & 35,9 & 116 & 31,6 & 212 & $1,75(1,07-2,90)$ & 0,0259 \\
\hline $30-34$ & 25,1 & 81 & 28,3 & 190 & $1,37(0,81-2,30)$ & 0,2590 \\
\hline 35 e mais & 10,5 & 29 & 17,3 & 93 & 1 & \\
\hline \multicolumn{7}{|l|}{ Laqueadura tubária } \\
\hline Sim & 70,2 & 261 & 68,2 & 505 & $1,09(0,83-1,45)$ & 0,5597 \\
\hline Não & 29,8 & 111 & 31,8 & 235 & 1 & \\
\hline \multicolumn{7}{|l|}{ Contraceptivo oral2 } \\
\hline $\operatorname{Sim}$ & 63,0 & 225 & 62,6 & 451 & $1,02(0,78-1,33)$ & 0,9549 \\
\hline Não & 37,0 & 132 & 37,4 & 269 & 1 & \\
\hline \multicolumn{7}{|l|}{ Uso do DIU2 } \\
\hline Sim & 1,4 & 5 & 3,2 & 23 & $0,43(0,16-1,14)$ & 0,1030 \\
\hline Não & 98,6 & 352 & 96,8 & 696 & 1 & \\
\hline
\end{tabular}

1 Categoria de referência.

2 Uso atual ou no passado.

$\mathrm{OR}=$ odds ratio. 
Características selecionadas dos casos de histerectomia e controles segundo a paridade (número de filhos tidos).

\begin{tabular}{|c|c|c|c|c|c|c|}
\hline \multirow[t]{3}{*}{ Características } & \multicolumn{6}{|c|}{ Paridade (\%) } \\
\hline & \multicolumn{3}{|c|}{ Casos } & \multicolumn{3}{|c|}{ Controles } \\
\hline & $\begin{array}{c}\text { Nenhum } \\
(n=54)\end{array}$ & $\begin{array}{c}1 \text { a } 3 \\
(n=209)\end{array}$ & $\begin{array}{l}4 \text { e mais } \\
(n=110)\end{array}$ & $\begin{array}{l}\text { Nenhum } \\
(n=66)\end{array}$ & $\begin{array}{c}1 \text { a } 3 \\
(n=393)\end{array}$ & $\begin{array}{c}4 \text { e mais } \\
(n=283)\end{array}$ \\
\hline Idade inferior a 40 anos & 39,2 & 35,6 & 12,9 & 39,6 & 39,4 & 14,6 \\
\hline Renda familiar inferior à medianal & 19,6 & 33,7 & 51,5 & 41,7 & 47,8 & 71,2 \\
\hline Nunca viveu em união & 54,7 & 3,3 & 0,0 & 55,4 & 1,0 & 0,7 \\
\hline Sem companheiro & 66,7 & 22,5 & 25,5 & 73,8 & 25,6 & 26,9 \\
\hline Sem vida sexual ativa & 66,7 & 22,5 & 22,9 & 72,3 & 27,6 & 31,4 \\
\hline N unca usou contracepção & 60,5 & 7,7 & 1,8 & 55,8 & 8,9 & 5,0 \\
\hline Refere "dificuldade" $p /$ engravidar & 25,8 & 11,9 & 0,9 & 37,1 & 13,5 & 5,4 \\
\hline Número de gestações & $87 \%=0$ & $50 \%$ Ž 3 & $50 \%$ Ž 5 & $90,8 \%=0$ & $50 \%$ Ž 3 & $50 \%$ Ž 6 \\
\hline Com história de aborto & 13,0 & 38,8 & 31,8 & 9,2 & 36,0 & 44,9 \\
\hline Nunca teve parto cirúrgico & 0,0 & 50,7 & 49,1 & 0,0 & 48,5 & 59,4 \\
\hline Fez laqueadura tubária & 0,0 & 77,0 & 91,7 & 0,0 & 69,7 & 81,9 \\
\hline Está na menopausa & 5,6 & 3,8 & 3,6 & 18,5 & 13,7 & 18,4 \\
\hline
\end{tabular}

1 A mediana da renda familiar mensal per capita mensal foi igual a $\mathrm{R} \$ 68,00$ (meio salário mínimo).

Posteriormente, as variáveis que permaneceram no modelo biológico e no motivacional foram reunidas em um modelo único. Todas as variáveis presentes no modelo final são apresentadas na Tabela 5. Ao final da análise foram confirmados como fatores de risco para histerectomia: a renda familiar per capita relativamente maior, a nuliparidade e a paridade de até três filhos, a demanda por cuidados médicos por problemas menstruais, o antecedente de distúrbio menstrual ou de morbidade referida do colo uterino, a hospitalização por causa ginecológica e a presença de laqueadura tubária antes dos trinta anos. A história prévia de filho nascido morto e a menopausa se constituíram em fatores de proteção.

\section{Discussão}

Este estudo vem contribuir para conferir visibilidade à prática da histerectomia no Brasil, que, como mostra a literatura internacional, vem se constituindo em um novo elemento no processo de medicalização do corpo feminino.

As mulheres submetidas à histerectomia eram relativamente jovens. O aumento do risco de histerectomia com a idade das mulheres tem sido relatado em outros estudos (Luoto et al., 1997; Settnes \& Jorgensen, 1996), ainda que não seja bem compreendido se este fato resultaria do aumento, com a idade, de condições ou enfermidades ginecológicas ou do favoreci- mento de soluções cirúrgicas, em decorrência da concepção médica de que a preservação do útero se faz necessária unicamente enquanto mantida a função reprodutiva (Goldhaber et al., 1993). É possível que essa distribuição etária dos casos reflita a intensificação do processo de medicalização entre as coortes de mulheres mais jovens, acrescido do fato de que o término da vida reprodutiva vem ocorrendo em idade mais jovem (BEMFAM/DHS, 1997).

Dentre as características sócio-demográficas estudadas, apenas a renda familiar mensal per capita mostrou-se associada ao risco de histerectomia, a despeito de tratar-se de clientela do setor público de saúde. A associação positiva entre a situação sócio-econômica e o risco de histerectomia foi também relatada em outras pesquisas (Luoto et al., 1997; Settnes \& Jorgensen, 1996). Isso provavelmente decorre do acesso desigual à investigação clínica e aos procedimentos terapêuticos, inclusive cirúrgicos, mesmo em situação de pobreza e em segmentos da população com nível de renda aparentemente tão homogêneo como as mulheres entrevistadas.

Em contraste com estudos realizados em outros países, que relataram um aumento progressivo do risco de histerectomia com a elevação do número de filhos (Brett et al., 1997; Santow \& Bracher, 1992; Settnes \& Jorgensen, 1996), observou-se um acréscimo do risco na ausência de paridade e entre as mulheres que tiveram até três filhos, tendo-se como grupo de compa- 
Características dos casos de histerectomia e controles segundo história menstrual, de morbidade ginecológica e uso de serviços de saúde.

\begin{tabular}{|c|c|c|c|c|c|c|}
\hline \multirow[t]{2}{*}{ Características } & \multicolumn{2}{|c|}{ Casos } & \multicolumn{2}{|c|}{ Controles } & \multicolumn{2}{|c|}{ OR bruta } \\
\hline & $\%$ & $\mathrm{n}$ & $\%$ & $\mathrm{n}$ & OR (IC95\%) & valor-p \\
\hline \multicolumn{7}{|l|}{ Idade na menarca (anos) } \\
\hline $8-13$ & 64,7 & 238 & 61,2 & 436 & $1,16(0,89-1,50)$ & 0,2690 \\
\hline $14 \mathrm{e}+$ & 35,3 & 130 & 38,8 & 276 & 1 & - \\
\hline \multicolumn{7}{|l|}{$\begin{array}{l}\text { Padrão menstrual prevalente } \\
\text { aos vinte anos }\end{array}$} \\
\hline Com transtorno menstrual & 8,1 & 30 & 2,3 & 17 & $3,75(2,04-6,89)$ & 0,0000 \\
\hline Sem transtorno menstrual & 91,9 & 340 & 97,7 & 722 & - & - \\
\hline \multicolumn{7}{|c|}{$\begin{array}{l}\text { Consultou médico por problemas } \\
\text { menstruais }\end{array}$} \\
\hline Procurou alguma vez & 31,8 & 118 & 10,8 & 80 & $3,85(2,80-5,30)$ & 0,0000 \\
\hline Nunca procurou & 68,2 & 253 & 89,2 & 661 & 1 & \\
\hline \multicolumn{7}{|l|}{$\begin{array}{l}\text { Padrão menstrual prevalente } \\
\text { nos últimos } 5 \text { anos } 2\end{array}$} \\
\hline Com transtorno menstrual & 95,5 & 341 & 47,1 & 293 & $23,93(14,82-40,47)$ & 0,0000 \\
\hline Sem transtorno menstrual & 4,5 & 16 & 52,9 & 329 & - & - \\
\hline \multicolumn{7}{|l|}{ Menopausa ( $\mathrm{Não/Sim)}$} \\
\hline Não & 96,0 & 357 & 84,1 & 624 & $0,22(0,13-0,39)$ & 0,0000 \\
\hline Sim & 4,0 & 15 & 15,9 & 118 & 1 & \\
\hline $\begin{array}{l}\text { Doenças sexualmente } \\
\text { transmissíveis (Sim/Não) }\end{array}$ & 7,6 & 28 & 8,4 & 62 & $0,89(0,56-1,43)$ & 0,6500 \\
\hline $\begin{array}{l}\text { História de morbidade } \\
\text { do colo uterino (Sim/Não) }\end{array}$ & 41,5 & 152 & 31,0 & 228 & $1,58(1,22-2,05)$ & 0,0010 \\
\hline \multicolumn{7}{|l|}{$\begin{array}{l}\text { Hospitalização por causa } \\
\text { ginecológica (Sim/Não) }\end{array}$} \\
\hline $\operatorname{Sim}$ & 30,3 & 112 & 17,3 & 128 & $2,08(1,56-2,79)$ & 0,0000 \\
\hline Não & 69,7 & 259 & 82,7 & 614 & - & - \\
\hline \multicolumn{7}{|c|}{ Cirurgias ginecológicas prévias 3} \\
\hline 1 e mais & 27,1 & 101 & 17.5 & 130 & $1,75(1,30-2,5)$ & 0,0002 \\
\hline Nenhuma & 72,9 & 272 & 82,5 & 612 & 1 & - \\
\hline \multicolumn{7}{|c|}{ Número de consultas ginecológicas 4} \\
\hline 4 e mais & 60,3 & 214 & 27,4 & 200 & $4,02(3,05-5,31)$ & 0,0000 \\
\hline Até 35 & 39,7 & 141 & 72,6 & 530 & - & - \\
\hline \multicolumn{7}{|c|}{ Número de ginecologistas consultados 4} \\
\hline Até 15 & 44,6 & 157 & 70,9 & 517 & $3,03(2,33-3,94)$ & 0,0000 \\
\hline 2 e mais & 55,4 & 195 & 29,1 & 212 & 1 & - \\
\hline
\end{tabular}

1 Categoria de referência.

2 Excluídas as mulheres que não menstruavam no último ano,

tem como referência o padrão menstrual prevalente aos 20 anos.

3 Excluídas as laqueaduras tubárias e as cesarianas.

4 Tem como perío do de referência os três anos anteriores à entrevista.

5 18,8\% (137) controles não realizaram consulta ginecológica nos últimos três anos.

$O R=$ odds ratio. 
Fatores de risco para histerectomia, odds ratio $(\mathrm{OR})$, ajustada pelas variáveis presentes no modelo, intervalo de confiança de $95 \%$ (IC 95\%), valores de p correspondentes.

\begin{tabular}{|c|c|c|c|}
\hline Variáveis & OR & IC95\% & $\mathrm{p}$-valor \\
\hline \multicolumn{4}{|l|}{ Modelo biológico } \\
\hline Idade (em anos) & 1,00 & $0,97-1,02$ & 0,9349 \\
\hline Gravidez (Sim/Não) & 1,84 & $1,18-2,87$ & 0,0075 \\
\hline Filhos nascidos mortos (Sim/Não) & 0,43 & $0,23-0,78$ & 0,0059 \\
\hline Distúrbios menstruais aos vinte anos (Sim/Não) & 3,36 & $1,75-6,43$ & 0,0003 \\
\hline Cuidados médicos por problemas menstruais(Sim/Não) & 3,38 & $2,40-4,78$ & 0,0000 \\
\hline Hospitalização por causa ginecológica (Sim/Não) & 1,47 & $1,06-2,43$ & 0,0206 \\
\hline Antecedente de enfermidade do colo uterino (Sim/Não) & 1,61 & $1,22-2,14$ & 0,0009 \\
\hline \multicolumn{4}{|l|}{ Modelo motivacional } \\
\hline Idade (em anos) & 1,06 & $1,03-1,08$ & 0,0001 \\
\hline \multicolumn{4}{|l|}{ Paridade (número/4 e mais) } \\
\hline Nulípara & 3,18 & $1,83-0,53$ & 0,0000 \\
\hline $1-3$ partos & 1,41 & $1,03-0,94$ & 0,0335 \\
\hline Menopausa (Sim/Não) & 0,17 & $0,09-0,31$ & 0,0000 \\
\hline Renda familiar ( $50 \%$ menos $/ 50 \%$ mais pobres) & 1,68 & $1,28-2,20$ & 0,0002 \\
\hline Laqueadura tubária & & & 0,0027 \\
\hline Antes dos 30 anos & 1,88 & $1,28-2,76$ & 0,0012 \\
\hline Aos 30 anos ou mais & 1,25 & $0,83-1,88$ & 0,2765 \\
\hline \multicolumn{4}{|l|}{ Modelo final } \\
\hline Idade (em anos) & 1,05 & $1,02-1,08$ & 0,0005 \\
\hline Paridade & & & 0,0007 \\
\hline Nulípara (nenhum/4 e mais) & 3,16 & $1,74-5,71$ & 0,0001 \\
\hline $1-3$ filhos ( 1 a $3 / 4$ e mais) & 1,50 & $1,06-2,10$ & 0,0210 \\
\hline Menopausa (Sim/Não) & 0,18 & $0,10-0,33$ & 0,0000 \\
\hline Filhos nascidos mortos (Sim/Não) & 0,51 & $0,27-0,95$ & 0,0344 \\
\hline Renda familiar ( $50 \%$ menos $/ 50 \%$ mais pobres) & 1,59 & $1,20-2,11$ & 0,0014 \\
\hline Distúrbios menstruais aos vinte anos (Sim/Não) & 3,29 & $1,66-6,48$ & 0,0006 \\
\hline Laqueadura tubária & & & 0,0498 \\
\hline Antes dos 30 anos & 1,54 & $1,03-2,30$ & 0,0351 \\
\hline Aos 30 anos ou mais & 1,07 & $0,70-1,64$ & 0,7544 \\
\hline Cuidados médicos por problemas menstruais (Sim/Não) & 3,51 & $2,43-5,06$ & 0,0000 \\
\hline Antecedente de enfermidade do colo uterino (Sim/Não) & 1,48 & $1,10-1,98$ & 0,0095 \\
\hline Hospitalização por causa ginecológica (Sim/Não) & 1,40 & $0,99-1,97$ & 0,0568 \\
\hline
\end{tabular}

ração aquelas com paridade mais elevada. Este perfil a princípio parece paradoxal, entretanto, sugere que a paridade não estaria expressando o efeito de processos unicamente biológicos relacionados à gravidez, mas refletindo outras dimensões da vida das mulheres que seriam determinantes do risco de histerectomia. De fato, os três níveis de paridade representam três perfis muito distintos de mulheres. Assim, dentre as que nunca tiveram filhos predominam aquelas com condições sócio-econômicas relativamente melhores, as quais ao mesmo tempo, agregam outras características que no senso comum de- marcam a não concretização de uma identidade de "mulher", seja por nunca ter tido companheiro ou vida sexual, por nunca ter vivido em união, nunca ter engravidado ou parido. Entre aquelas com paridade mais reduzida (um a três filhos), predominam as mais jovens e com história reprodutiva relativamente mais medicalizada. Por último, entre aquelas com maior paridade há o predomínio de mulheres com idade mais elevada, que vivem em moldes mais tradicionais e em piores condições de vida.

A associação entre histerectomia e laqueadura tubária tem sido documentada, de forma 
recorrente, em outros estudos (Goldhaber et al., 1993; Hillis et al., 1997; Stergachis et al., 1990), e à semelhança do que foi constatado no presente estudo, sendo o risco mais elevado para as mulheres que realizaram esse procedimento quando mais jovens (Sterggachis et al., 1990). No entanto, persiste a ausência de consenso quanto à existência de uma fundamentação biológica para o excesso de histerectomias entre mulheres com laqueadura, pois se há evidências da maior incidência de distúrbios menstruais entre estas (Wilcox et al., 1992), também há pesquisas que não a confirmam (Rulin et al., 1993). Além disso, há algumas evidências de que a associação entre histerectomia e laqueadura tubária pode não refletir apenas fatos de natureza biológica, mas a conjugação entre os efeitos adversos do método e uma maior propensão dos médicos a optarem por uma solução cirúrgica para um problema ginecológico de natureza benigna ao tratar-se de uma mulher que já encerrou definitivamente sua vida reprodutiva (Goldhaber, 1993; Stergachis et al., 1990).

Entre os presentes achados surpreendeu a presença de uma relação inversa entre histerectomia e a história anterior de gravidez que resultou em natimorto, fato que diverge de outros estudos (Settnes \& Jorgensen, 1996). Na presença de achados positivos, discute-se em que medida essas histerectomias foram empreendidas com a finalidade de interromper histórias reprodutivas entrecortadas por problemas de saúde e perdas fetais (Settnes \& Jorgensen, 1996). Os resultados do presente estudo indicam que o antecedente de perdas fetais pode interferir em decisões cirúrgicas na direção oposta àquela, até então, documentada em outras pesquisas, o que seria interessante elucidar em investigações posteriores.

Nos resultados da pesquisa, apresentados em outro lugar (Barreto-de-Araújo, 2002), há evidências de que em alguns casos a indicação das histerectomias não foi baseada em critérios bem definidos. Para 5,9\% do total das indicações, ao exame histopatológico o útero estava inteiramente sadio ou as alterações descritas eram de natureza benigna e com localização no colo uterino. Adicionalmente, o diagnóstico inicial não foi confirmado em uma proporção de casos que variou de 2,9\% a 14,3\% entre os hospitais. Dentre as indicações feitas, predominou o diagnóstico de leiomioma uterino. Este diagnóstico, responsável pela quase totalidade das histerectomias, por consistir em tumor benigno, implica fundamentalmente o julgamento clínico quanto à necessidade da cirurgia, ainda quando sintomático (Myers et al., 2002).
Em estudos realizados em países da Europa e nos Estados Unidos, embora o leiomioma uterino predomine entre as indicações, este diagnóstico responde por uma proporção que varia de 30 a $60 \%$ das histerectomias (Settnes \& Jorgensen, 1996; Vessey et al., 1992; Wilcox et al., 1994). A diferença observada entre os casos aqui apresentados e os estudos citados, quanto à participação do leiomioma entre as indicações, pode representar uma menor participação das demais causas específicas que compõem o elenco de indicações descritas em outros locais, ou uma maior propensão dos médicos a indicar a histerectomia diante desse diagnóstico no contexto estudado.

Quando se observam os demais fatores associados à prática da histerectomia, estes remetem à história menstrual das mulheres, a qual abrange não só a existência de distúrbios da função menstrual em período imediatamente anterior à cirurgia, como também, os internamentos por causa ginecológica que de modo geral foram por sangramento uterino. Os distúrbios menstruais, apesar de terem sido relatados também com freqüência elevada pelas mulheres do grupo de comparação, foram efetivamente o principal motivo pelo qual as mulheres procuraram cuidados médicos, tendo assim, desencadeado o processo de investigação diagnóstica que resultou na indicação da histerectomia. A presença da menopausa integra este conjunto de variáveis, sendo provável que o efeito protetor evidenciado esteja mediado pela ausência de sangramento menstrual.

A trajetória até a histerectomia de uma mulher com problemas menstruais é complexa, considerando-se que vários percursos são possíveis. Primeiro, é preciso a mulher perceber as alterações menstruais como um problema; ao considerá-las como tal, decidir consultar um médico e conseguir ser atendida; em sendo diagnosticada uma enfermidade uterina benigna e indicada a cirurgia, é necessário aceitá-la e ter acesso ao procedimento. Mesmo assim, os resultados aqui apresentados podem sugerir a existência de uma tendência à realização de procedimentos cirúrgicos para resolução de demandas por enfermidades benignas, cujo manuseio não implica necessariamente a realização de cirurgia (Myers et al., 2002), a qual envolve riscos que seriam, portanto, desnecessários.

De modo geral, a indicação de histerectomia por doença benigna implica estabelecer a cirurgia como necessária, o que depende do julgamento médico construído com base em considerações quanto à gravidade dos sintomas e de uma série de outros aspectos mais subjetivos. 
No que diz respeito aos limites do estudo, o principal aspecto consiste na identificação dos controles com base no relato das mulheres sobre ter o útero preservado, e na obtenção de informações quanto às exposições por meio de entrevistas. No entanto, a remoção do útero implica o desaparecimento definitivo da menstruação, fato que não passaria desapercebido às mulheres. Além disso, há evidências de que é possível obter-se informações quanto à histerectomia, laqueadura tubária e outros aspectos da vida reprodutiva com elevado grau de

\section{Agradecimentos}

Esta pesquisa foi subvencionada parcialmente pelo Conselho Nacional de Desenvolvimento Científico e Tecnológico (Programa de Bolsas) e pela Fundação Ford (Doação no 9901033). acurácia, a partir da aplicação de questionários (Green et al., 1997). De qualquer modo, a eventual inclusão de mulheres sem o útero preservado entre os controles teria resultado na subestimação das associações.

Para concluir, as observações contidas neste artigo apontam a necessidade de outras investigações empíricas que permitam aprofundar o conhecimento dessa realidade em âmbito nacional, como também, das particularidades desta prática no cuidado à saúde das mulheres pertencentes a outros segmentos sociais.

\section{Referências}

BARRETO-DE-ARAÚJO, T. V., 2002. Esterilização Feminina por Laqueadura Tubária e Histerectomia Subseqüente: A Experiência das Mulheres Usuárias dos Serviços Públicos de Saúde em Recife, Pernambuco. Tese de Doutorado, Salvador: Instituto de Saúde Coletiva, Universidade Federal da Bahia. BEMFAM (Sociedade Civil Bem-estar Familiar no Brasil)/DHS (Demography and Health Survey), 1997. Pesquisa Nacional sobre Demografia e SaúdeBrasil-1996. Rio de Janeiro: BEMFAM/DHS.

BICKELL, N. A.; EARP, J. A.; GARRETT, J. M. \& EVANS, A. T., 1994. Gynecologists' sex, clinical beliefs, and hysterectomy rates. American Journal of Public Health, 84:1649-1651.

BRETT, K. M.; MARSH, J. V. \& MADANS, J. H., 1997. Epidemiology of hysterectomy in the United States: Demographic and reproductive factors in a nationally representative sample. Journal of Women's Health, 6:309-316.

DOMENIGHETTI, G.; LURASCHI, P.; CASABIANCA, A.; GUTZWILLER, F.; SPINELLI, A.; PEDRINIS, E. \& REPETTO, F., 1988. Effect of information campaign by the mass media on hysterectomy rates. Lancet, 2:1470-1473. 
GOLDHABER, M. K.; ARMSTRONG, M. A.; GOLDITCH, I. M.; SHEEHE, P. R.; PETITTI, D. B. \& FRIEDMAN, G. D., 1993. Long-term risk of hysterectomy among 80,007 sterilized and comparison women at Kaiser Permanente, 1971-1987. American Journal of Epidemiology, 130:508-521.

GREEN, A.; PURDIE, D.; GREEN, L.; DICK, M. L.; BAIN, C. \& SISKIND, V., 1997. Validity of self-reported hysterectomy and tubal sterilization - The Survey of Women's Health Study Group. Australian and New Zealand Journal of Public Health, 21:377-340.

HAAS, S.; ACKER, D. \& DONAHUE, C., 1993. Variation in hysterectomy rates across small geographic areas of Massachusetts. American Journal of Gynecology, 169:150-154.

HILLIS, S. D.; MARCHBANKS, P. A.; TYLOR, L. R. \& PETERSON, H. B., 1997. Tubal sterilization and longterm risk of hysterectomy: Findings from the United States Collaborative Review of Sterilization. The United States Collaborative Review of Sterilization Working Group. Obstetrics and Gynecology, 91:241-246.

LUOTO, R.; KESKIMAKI, I. \& REUNANEN, A., 1997. Socioeconomic variations in hysterectomy: Evidence from a linkage study of the Finnish hospital discharge register and population census. Journal of Epidemiology and Community Health, 51: 67-73.

DATASUS (Departamento de Informática do SUS), s.d. Sistema de Informações Hospitalares do SUS (SIH/ SUS): Banco de Dados. 7 Março $2002<\mathrm{http}$ // www.datasus.gov.br>.

MYERS, E. R.; BARBER, M. D.; ASHBY, T. G.; COUCHMAN, G.; MATCHAR, D. B. \& McCRORY, D., 2002. Management of uterine leiomyomata: What do we really know? Obstetrics and Gynecology, 100:8-17.

ROOS, N. P., 1984. Hysterectomy: Variations in rates across small areas and across physicians' practices. American Journal of Public Health, 74:39-46.
RULIN, M. C.; DAVIDSON, A. R.; PHILlLBER, S.G.; GRAVES, W. L. \& CUSHMAN, L. F., 1993. Longterm effect of tubal sterilization on menstrual indices and pelvic pain. Obstetrics and Gynecology, 82:118-121.

SANTOW, G. \& BRACHER, M., 1992. Correlates of hysterectomy in Australia. Social Science and Medicine, 34:929-942.

SETTNES, A. \& JORGENSEN, T., 1996. Hysterectomy in a Danish cohort. Prevalence, incidence and socio-demographic characteristics. Acta Obstetricia et Gynecologica Scandinavica, 75:274-280.

STERGACHIS, A.; SHY, K. K.; GROTHAUS, L. C.; WAGNER, E. H.; HECHT, J. A.; ANDERSON, G.; NORMAND, E. H. \& RABOUD, J., 1990. Tubal sterilization and the long term risk of hysterectomy. $J A$ MA, 264:2893-2898.

TRELOAR, A. S.; DO, K. A.; O'CONNOR, V. M.; O'CONNOR, D. T.; YEO, M. A. \& MARTIN, N. G., 1999. Predictors of hysterectomy: An Australian study. American Journal of Obstetrics and Gynecology, 160:945-954.

VESSEY, M. P.; VILLARD-MACKINTOSH, L.; McPHERSON, K.; COULTER, A. \& YEATES, D., 1992. The epidemiology of hysterectomy: Findings in a large cohort study. British Journal of Obstetrics and Gynaecology, 99:402-407.

WILCOX, L. S.; MARTINEZ-SCHNELL, B.; PETERSON, H. B.; WARE, J. H. \& HUGHES, J. M., 1992. Menstrual function after tubal sterilization. American Journal of Epidemiology, 135:1368-1381.

WILCOX, L. S.; KOONIN, L. M.; POKRAS, R.; STRAUSS, L. T.; XIA, Z. \& PETERSON, H. B., 1994. Hysterectomy in the United States, 1988-1990. Obstetrics and Gynaecology, 83:549-555.

Recebido em 18 de setembro de 2003

Versão final reapresentada em 19 de novembro de 2003 Aprovado em 28 de novembro de 2003 